\title{
Measuring helium abundance difference in giants of NGC $2808^{\star}$
}

\author{
L. Pasquini ${ }^{1}$, P. Mauas ${ }^{2}$, H. U. Käuf ${ }^{1}$, and C. Cacciari ${ }^{3}$ \\ 1 ESO - European Southern Observatory, Karl-Schwarzschild-Strasse 2, 85748 Garching bei München, Germany \\ e-mail: lpasquin@eso.org \\ 2 IAFE, CONICET-UBA and FCEN, UBA, Ciudad Universitaria, Buenos Aires, Argentina \\ 3 Istituto Nazionale di Astrofisica, Osservatorio Astronomico di Bologna, via Ranzani 1, 40127 Bologna, Italy
}

Received 26 January 2011 / Accepted 21 April 2011

\section{ABSTRACT}

\begin{abstract}
Context. Multiple populations have been detected in several globular clusters (GC) that do not display a spread in metallicity. Unusual features of their observed colour-magnitude diagrams (CMD) can be interpreted in terms of differences in the helium content of the stars belonging to the sub-populations.

Aims. Even if evidence gathered so far is compelling, differences in He abundance have never been directly observed. We attempt to measure these differences in two giant stars of NGC 2808 with very similar astrophysical parameters but different Na and O abundances, hence that presumably belong to different sub-populations, by directly comparing their He I $10830 \AA ̊$ lines.

Methods. The He $10830 \AA$ line forms in the upper chromosphere. Our detailed models derive the chromospheric structure using the $\mathrm{Ca}$ II and $\mathrm{H} \alpha$ chromospheric lines, and simulate the corresponding He I 10830 line profiles as a function of the helium abundance. We show that, at a given value of He abundance, the He I 10830 equivalent width cannot significantly change without a corresponding much larger change in the Ca II chromospheric lines. We have used the VLT-CRIRES to obtain high-resolution spectra in the $10830 \AA$ A region, and the VLT-UVES to obtain spectra of the Ca II and $\mathrm{H} \alpha$ lines of our target stars.

Results. The two target stars have very similar Ca II and $\mathrm{H} \alpha$ chromospheric lines, but different appearances in the He region. One line, blueshifted by $17 \mathrm{~km} \mathrm{~s}^{-1}$ with respect to the He 10830 rest wavelength, is detected in the spectrum of the Na-rich star, whereas the Na-poor star spectrum is consistent with a non-detection. From a detailed chromospheric modeling, we show that the difference in the spectra is consistent and most closely explained by an He abundance difference between the two stars of $\Delta Y \geq 0.17$. Our optical observations bracket the infrared ones over a range of about 50 days and we do not observe any substantial variability in the Ca II and $\mathrm{H} \alpha$ lines.

Conclusions. We provide direct evidence of a significant He line strength difference in giant stars of NGC 2808 belonging to different sub-populations, which had been previously detected by other photometric and spectroscopic means. The use of appropriate model chromospheres allows us for the first time to provide an approximate quantitative estimate of this difference, which is clearly consistent with the expected difference in abundance required by the stellar evolution theory to account for the observed peculiarities of this cluster's cmD.
\end{abstract}

Key words. stars: abundances - globular clusters: general - globular clusters: individual: NGC 2808

\section{Introduction}

Light element ( $\mathrm{CNO}$ cycle elements, $\mathrm{Mg}, \mathrm{Na}$ ) variations and anti-correlations in globular cluster (GC) red giant branch (RGB) stars have been known to exist for nearly 20 years (see e.g. Carretta et al. 2010, for a review) and for a long time were thought to be caused by mixing phenomena during the RGB phase. The detection of an abundance spread and element correlations in main sequence stars demonstrated that they are the result of pollution from previous generations of stars that contaminated the original cluster gas with $\mathrm{CNO}$ (and $\mathrm{Na}-\mathrm{Mg}$ ) cycled material (Gratton et al. 2001).

A vigorous discussion is ongoing about the nature of the stars producing these chemical anomalies, the most quoted candidates being either high mass stars (Decressin et al. 2007) or intermediate mass AGB's (D’Antona \& Caloi 2008). Two conclusions common to all scenarios are that a large amount of He was produced and that the polluted stars must be He enriched.

\footnotetext{
* Based on observations collected at ESO VLT, Chile, under programme 384.D-0283.
}

The discovery of multiple main sequences in several GCs, which can be accounted for by a large (sometimes huge) difference in He abundance among the different populations, is fully consistent with this scenario. In $\omega$ Cen, where multiple main sequences were firstly observed, the blue main-sequence stars must have a higher He abundance to explain their higher metallicity (Piotto et al. 2005). A perhaps more remarkable result is that multiple main sequences are also observed in NGC 2808 and in other GCs without any significant heavy element abundance spread (Piotto et al. 2007). According to stellar evolution prescriptions, large differences in He abundance are needed to account for these multiple populations. In NGC 2808, for example, a detailed photometric and spectroscopic study of RGB bump stars requires $\mathrm{He}$ abundance differences spanning a range of $\Delta Y$ from 0.11 to 0.19 among the three sub-populations identified in this cluster (Bragaglia et al. 2010). A similar difference, $\Delta Y \sim 0.15$, is proposed by D'Antona \& Caloi (2008) to account for its horizontal branch (HB) morphology. From the detailed analysis of several GCs by Gratton et al. (2010), He is most likely to be the third parameter, along with metallicity and age, affecting the HB morphology. 


\section{He abundance variations as diagnostics of multiple populations in GCs}

\subsection{The He 10830 line: observations}

The evidence that $\mathrm{He}$ is enhanced in the "polluted" secondgeneration stars, is strong but so far only circumstantial.

The main obstacle to a direct spectroscopic confirmation is that He is hardly observable in cool stars, because no photospheric He lines are present in their atmospheres. Helium lines are observable in hot HB stars of GCs, where however their appearance is dominated by other effects, such as diffusion, that prevent reliable estimate of abundances (e.g. Mohler et al. 2007). The only He lines observable in cool stars are the $10830 \AA$ (and in some cases the fainter $5876 \AA$, cf. Danks \& Lambert 1985) chromospheric lines.

The $10830 \AA$ line (hereafter He 10830) has a chromospheric component that forms in the upper chromosphere of a low-gravity cool star farther out than either the $\mathrm{H} \alpha$ absorption core or the $\mathrm{Ca}$ II and $\mathrm{Mg}$ II emission cores. The line profile can provide insight into the dynamics of the upper atmosphere, where a wind begins to accelerate, hence it has been used with to study mass loss and chromospheric activity in several field metal-poor red giants and in a few RGB stars in the GC M13 (Dupree et al. 1992; Smith et al. 2004; Dupree et al. 2009). In these studies the line was shown to be detectable in stars fainter than $M_{v} \sim-1.5 \mathrm{mag}$ and hotter than $\sim 4500 \mathrm{~K}$. Dupree et al. (2011) observed the He 10830 line in twelve $\omega$ Cen giant stars belonging to different sub-populations, and found variations in the equivalent width as a function of the $\mathrm{Al}$ and $\mathrm{Na}$ abundances, and perhaps also of metallicity. They could not, however, quantify these differences in terms of He abundance between the different stars.

Earlier observations of the He 10830 line in field stars include the large surveys of Zirin (1982) and O'Brien \& Lambert (1986). The latter work is particularly interesting because the authors study in detail giants of different spectral types and the time variability of the line over several timescales.

\subsection{The He 10830 line: simulations}

The He 10830 line is actually a triplet spanning the range 9231.8565-9230.792 $\mathrm{cm}^{-1}$ (vacuum wavenumbers), the strongest member of the multiplet occurring at $9230.792 \mathrm{~cm}^{-1}$. It forms in non-LTE conditions, in the upper chromosphere above $10^{4} \mathrm{~K}$; therefore, it is difficult to use this line to determine the stellar He abundance in cool stars, hence it has never been previously used for this purpose. However, we show that relative He abundances can be estimated with the help of adequate chromosphere models.

We modelled the He 10830 line in our target stars based on our previous work in NGC 2808 giants (Cacciari et al. 2004), using the $\mathrm{Ca}$ II and $\mathrm{H} \alpha$ lines to model the chromospheres (Mauas et al. 2006). From these studies, it was clear that RGB stars in NGC 2808 have structured chromospheres, with plasma as hot as several thousands $\mathrm{K}$ and, at least for the most luminous objects, sufficiently fast velocity fields to produce mass loss along the RGB. We therefore expect the He 10830 line to be blue-shifted by several tens of $\mathrm{km} \mathrm{s}^{-1}$ because of the outward velocity field, and to be broadened because of the high temperature.

The purpose of these simulations is to investigate how strongly the He 10830 line reacts to variations in the He abundance and to variations in the chromospheric structure, to evaluate the extent to which the line can be used to distinguish

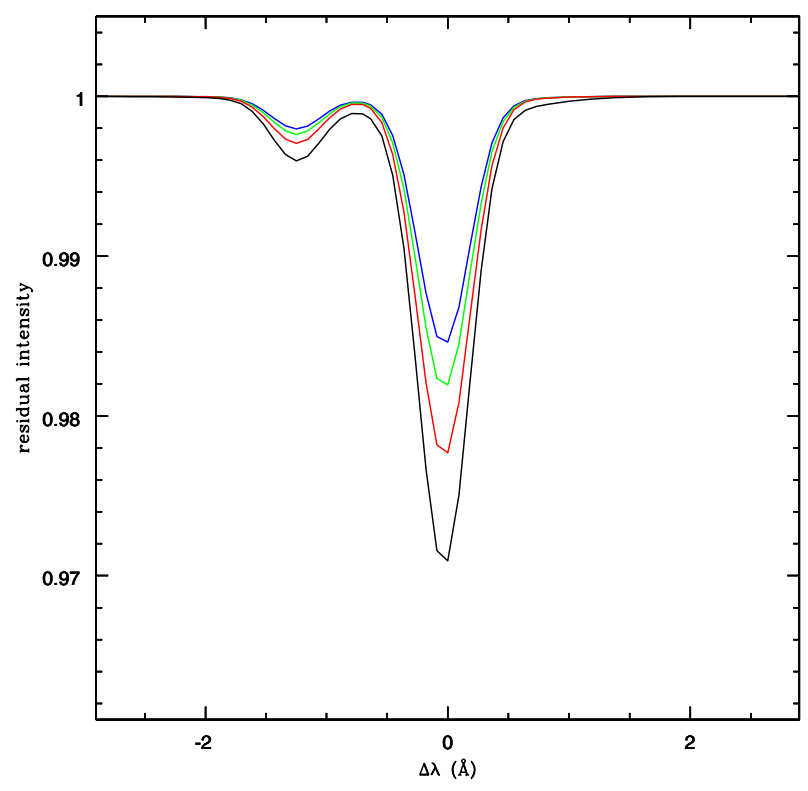

Fig. 1. Simulations of the He 10830 lines for our stars, using the chromospheric model of Mauas et al. (2006). The line equivalent width changes dramatically with the He abundance: the black line corresponds to $Y=0.3$, and the other lines correspond to a progressive decrease in steps of 0.03 in the He abundance.
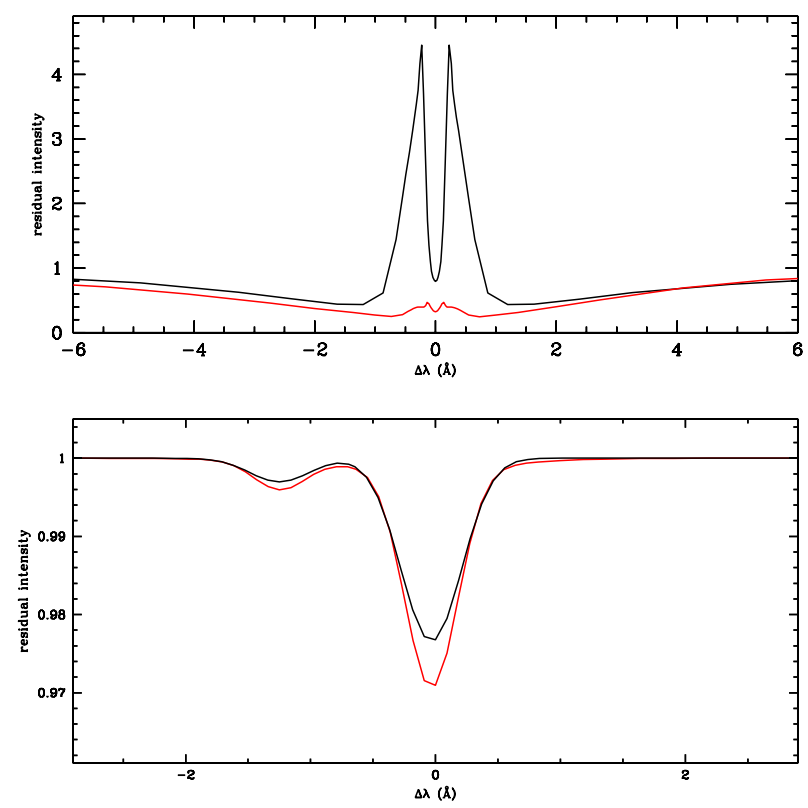

Fig. 2. Simulations of the He 10830 line (lower) and of the Ca II K (upper) line cores for star 48889 . The change in the He line strength (corresponding to a lower He abundance by $\Delta Y=-0.03$ ) is obtained by changing the chromospheric model only. As a consequence, the emission in the core of the $\mathrm{Ca}$ II line is greatly enhanced, as shown in the upper panel.

between these two effects. Figure 1 shows the He 10830 line profiles obtained with our model of NGC 2808 bright giants (star 48 889, Mauas et al. 2006) when varying the content of $\mathrm{He}$ in the atmosphere. The black line assumes a He abundance $Y=0.3$, and the other lines are computed by decreasing $Y$ progressively in steps of $10 \%$.

We also simulate the change in the chromospheric structure needed to produce the same effect on the He line profile as a $10 \%$ lower $Y$ (see Fig. 2). The He 10830 line forms in higher layers 
Table 1. Target stars. Astrophysical parameters and log of observations.

\begin{tabular}{lccccccccc}
\hline \hline Star name & $T_{\text {eff }}$ & $\log g$ & $M_{v}$ & {$[\mathrm{Fe} / \mathrm{H}]$} & {$[\mathrm{Na} / \mathrm{Fe}]$} & {$[\mathrm{O} / \mathrm{FE}]$} & $\begin{array}{c}\mathrm{RV} \\
\mathrm{km} \mathrm{s}^{-1}\end{array}$ & $\begin{array}{c}\text { Obs. UVES } \\
\text { int. time }=1200 \mathrm{~s}\end{array}$ & $\begin{array}{c}\text { Obs. CRIRES } \\
\text { int. time }=1800 \mathrm{~s}\end{array}$ \\
\hline 48889 & 3843 & 0.52 & -2.25 & $-1.22 \pm 0.12$ & +0.64 & -0.07 & 115.1 & $06 / 11 / 09 \& 26 / 12 / 09$ & $26 / 11 / 09$ \\
46422 & 3843 & 0.42 & -2.21 & $-1.08 \pm 0.09$ & +0.12 & +0.23 & 93.5 & $06 / 11 / 09 \& 02 / 12 / 09$ & $26 / 11 / 09$ \\
\hline
\end{tabular}

Notes. Stellar identification and astrophysical parameter values are from Cacciari et al. (2004) and Carretta et al. (2004). The [Fe/H] quoted are the values obtained from FeII.

than the $\mathrm{Ca}$ II $\mathrm{K}$ line. However, since it is dominated by radiation, its source function is decoupled from the Planck function in the region where the line is formed, and it depends on the conditions at inner layers. As we already pointed out in Mauas et al. (2006), "the computed profiles are unaffected by the structure of the regions where the temperature is higher than $10000 \mathrm{~K}$ ". Therefore, to mimic the lower He abundance profile the chromosphere has to be heated at layers where $T \lesssim 8000 \mathrm{~K}$. This, however, produces a huge variation on the profiles of the other chromospheric lines, e.g. the $\mathrm{K}$ line that is dominated by collisions, and its core reflects the temperature increase much more strongly. This variation would be readily observable, as is clearly shown in the upper panel of Fig. 2, where the Ca II K line cores obtained with the two chromospheric models are compared.

This does not imply that we are able to derive the He abundance from the observations of the He 10830 line and its modelling using the $\mathrm{Ca}$ II and $\mathrm{H} \alpha$ lines. However, for two similar metal-poor cool giants, with not only the same main physical parameters, but also a similar chromospheric structure, as for our twin target stars, it is possible to distinguish between changes in the He lines caused by different He abundances or by different chromospheres, because chromospheric changes large enough to produce a visible difference in the He line profile would also produce huge differences in the other chromospheric lines. We discuss this point further in Sect. 4.

\section{Observations and analysis}

We selected from the list of Carretta et al. (2004) two RGB stars in the cluster NGC 2808, with very similar stellar parameters but very different $\mathrm{Na}$ and $\mathrm{O}$ abundances (see Table 1). The Na-rich $\mathrm{O}$-poor star has been polluted by processed gas from a previous generation of stars and is expected to be representative of the He-enhanced population. The other star, with standard $\mathrm{O}$ and $\mathrm{Na}$ values, is taken to be representative of the unpolluted Henormal (primordial) population.

The observations were carried out in service mode at the ESO VLT. The UVES spectrograph (Dekker et al. 2000) was used to observe the stars in the $\mathrm{Ca}$ II $\mathrm{H}$ and $\mathrm{K}$ lines and in $\mathrm{H} \alpha$ at $R \sim 40000$. The IR high resolution spectrograph CRIRES (Käufl et al. 2004) was used for the He lines, with a similar resolution.

Complete information about the target stars and the observations is contained in Table 1 . The temperature values are those based on $B-V$ because no $V-K$ colour is available for star 46422 . The two stars have the same parameter values in the spectroscopic analysis of Carretta et al. (2004), but the spectroscopic temperatures are $100 \mathrm{~K}$ higher than the values based on the $B-V$ colours. Our stars are slightly brighter than $M_{V}=-1.5 \mathrm{mag}$ and cooler than $4600 \mathrm{~K}$, which represent the He 10830 line detection thresholds found by Smith et al. (2004) and Dupree et al. (2009), so it was not quite clear whether the He line would be detectable in the normal abundance star. On the other hand, these stars are bright enough to permit a spectrum of the appropriate signal-to-noise ratio $(S / N)$ to be obtained in a reasonably short integration time.

The spectra were processed starting from the output of the ESO pipelines. The UVES spectra taken in two separate runs (early November and December) are very similar for each star, therefore the average spectrum was used. We also observed a pair of fainter and hotter stars of NGC 2808, but the $S / N$ of the extracted CRIRES spectra was not high enough to permit a reliable analysis; improvements will require the modifications of the pipeline extraction software. The expected depth of the He line from our models is fairly small, therefore, even if the $S / N$ in the continuum of the stars is around 100 for a $0.044 \mathrm{~cm}^{-1}$ pixel, for the two brightest stars a special care was taken and the output spectra of the CRIRES pipeline (Jung \& Bristow 2008) were further treated; all spectra were deglitched and normalized to unity for the continuum using a low order polynomial.

The region around the He 10830 lines contains two telluric lines, a strong one at $9229.284 \mathrm{~cm}^{-1}$, and a weaker one (about $1 / 3$ of the equivalent width) at $9227.689 \mathrm{~cm}^{-1}$ (Breckinridge \& Hall 1973). The presence of these telluric lines can endanger a correct detection or measurement of the He 10830 lines. We therefore observed a rapidly rotating hot star for telluric correction (HD 79041, B6V, $v \sin (i)=248 \mathrm{~km} \mathrm{~s}^{-1}$ ), at an airmass similar to the one of the cluster targets (1.5 for HD 79041, 1.7 for 48889 and 1.53 for 46422 ). The spectrum of the standard star was first normalized and scaled by the ratio of the airmasses, to properly reproduce the depth of the telluric lines. The program star spectra were divided by the scaled spectra of the standard star. Figures 3 and 4 show in black the spectra of the program stars and in red the spectra of the standard star before the division. In these figures, wavelength is expressed in $\mathrm{cm}^{-1}$, no velocity adjustment has been applied and blue is to the right. The reader can note in Figs. 3 and 4 that the telluric lines are redshifted by $\sim 0.5 \mathrm{~cm} \mathrm{~s}^{-1}$ with respect to their nominal wavenumber because of the imperfect wavelength calibration. When taking into account the radial velocity of the stars (cf. Table 1), the He 10830 lines are expected to be blueward of $9227.25 \mathrm{~cm}^{-1}$ for star 48889 and of $9227.92 \mathrm{~cm}^{-1}$ for star 46422 .

The last step consisted of correcting the program star spectra for radial velocity by matching the mcmath Solar FTS spectra using the Solar FTS atlases from Wallace et al. (1993) centred on the HeI line. A small adjustment to the wavelength scale was also required to best match the solar atlas. The finally reduced spectra at rest-frame wavenumber are shown in Fig. 5. The spectra of the two NGC 2808 stars, show the same features, with the exception of two lines: at $\sim 9227 \mathrm{~cm}^{-1}$, where a Na doublet is present in star 48889 and is much smaller in star 46422, thus confirming the much higher $\mathrm{Na}$ abundance of star 48889 ; and at $\sim 9231.32 \mathrm{~cm}^{-1}$, where a rather broad line is clearly present in the spectrum of star 48889 but is missing in the spectrum of star 46422. 


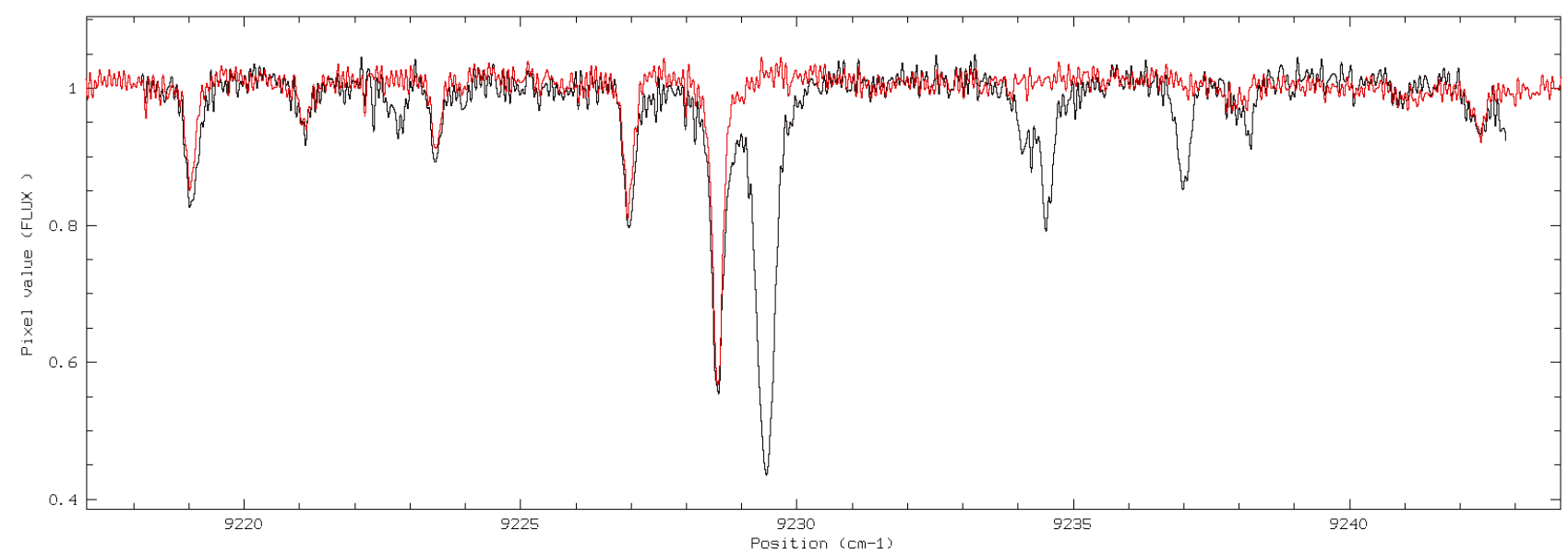

Fig. 3. Spectra of the star 48889 (black) and of the telluric standard star (red) before telluric correction. The abscissa is in $\mathrm{cm}^{-1}$, red is left. The wavelength calibration is from the CRIRES pipeline, without further adjustment, and shows a shift of about $0.5 \mathrm{~cm}^{-1}$ towards the red with respect to the nominal wavenumber of the telluric lines. The wavelength calibration and the correction for stellar radial velocity were performed in the last step of the data reduction, after telluric correction.

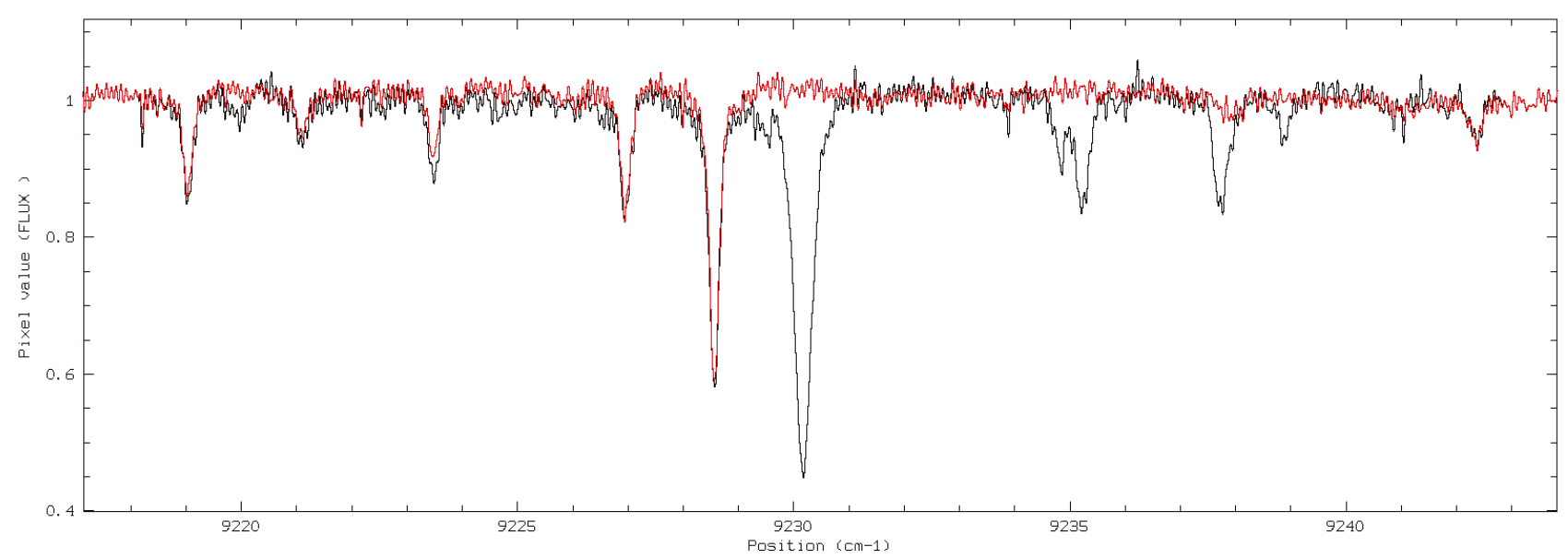

Fig. 4. Spectra of star 46422 (black), and of the telluric standard star (red) before telluric correction. The abscissa is in $\mathrm{cm}^{-1}$, red is left. The wavelength calibration is from the CRIRES pipeline, without further adjustment, and shows a shift of about $0.5 \mathrm{~cm}^{-1}$ towards the red with respect to the nominal wavenumber of the telluric lines. The wavelength calibration and the correction for stellar radial velocity has been performed in the last step of the data reduction, after telluric correction.

This feature has a different shape from the other lines: it is at least $17 \mathrm{~km} \mathrm{~s}^{-1}$ broad, and is blue-shifted by $\sim 17 \mathrm{~km} \mathrm{~s}^{-1}$ with respect to the rest wavelength of the strongest He 10830 component $\left(9230.792 \mathrm{~cm}^{-1}\right)$. This is approximately what is expected, in a few field stars the He lines have blue shifts larger than $60 \mathrm{~km} \mathrm{~s}^{-1}$ (Dupree et al. 2009), but most field giants have spectra strikingly similar to our spectrum of star 48 889. Several spectra presented in O'Brien \& Lambert (1986) also have He lines that are similar in depth and width to the one observed in star 48889 . The wavenumber of the nearby Si (9233.58) and $\mathrm{Na}$ (9226.94) lines are in excellent agreement with their vacuum measurements.

Since the expected (and the observed) He line is quite small (a few $\%$ of the continuum only), it is necessary to further develop some considerations about the significance of its detection. The $S / N$ in the continuum for these spectra is $S / N \sim 100$ for $0.044 \mathrm{~cm} \mathrm{~s}^{-1}$ pixel for both stars. For such a $S / N$, the error associated with the equivalent width of the He line is about $4 \mathrm{mcm}^{-1}$, or seven times smaller than the measured equivalent width (Cayrel 1988; see also discussion in Bonifacio et al. 2002). Apart from the contamination by the telluric line, there is, therefore no reason to question the reality and the detection of the He line. This is also confirmed by equivalent widths as small as a few $\mathrm{mcm}^{-1}$ being clearly observed and measured in the spectra (cf. Figs. 3-5 and Table 2). The only doubt that can therefore be reasonably cast concerns the blending of the telluric 9227.799 line with the He feature. However, as is clear from Fig. 3, the telluric and the He line are partially detached; in addition, we performed several tests changing the intensity of the telluric line, which did not substantially modify the equivalent width of the He line. We also note that the telluric lines have a width of $0.22 \mathrm{~cm} \mathrm{~s}^{-1}$, while the He line is at least $0.52 \mathrm{~cm} \mathrm{~s}^{-1}$ wide.

To avoid biases in our analysis, we asked a colleague to measure the equivalent width of the lines in the spectra and use an automatic spectral analysis program, DAOSPEC (Stetson \& Pancino 2008). Among other features, DAOSPEC defines its own continuum. A list of line identifications, measured wavenumbers, and equivalent widths for this region is given in Table 2. The equivalent widths are an average of the manual and the DAOSPEC measurements.

The largest difference between these measurements was about $6 \mathrm{mcm}^{-1}$, but for the strong Si line, for which the difference is up to $20 \mathrm{mcm}^{-1}$. DAOSPEC also estimates as well the errors in the equivalent widths, which are typically $1.5-2 \mathrm{mcm}^{-1}$, perfectly compatible with the measured differences. 
Table 2. Spectral lines in the spectral region around the He 10830 line.

\begin{tabular}{|c|c|c|c|c|c|c|}
\hline $\begin{array}{l}\text { Wavenumber (Meas.) } \\
\mathrm{cm}^{-1}\end{array}$ & $\begin{array}{c}\text { Wavenumber (Exp.) } \\
\mathrm{cm}^{-1}\end{array}$ & Element & $\begin{array}{c}\text { Width (48 889) } \\
\Delta \mathrm{cm}^{-1}\end{array}$ & $\begin{array}{c}\text { Width (46 422) } \\
\Delta \mathrm{cm}^{-1}\end{array}$ & $\begin{array}{c}\text { Eq. Width (48 889) } \\
\mathrm{mcm}^{-1}\end{array}$ & $\begin{array}{c}\text { Eq. Width (46 422) } \\
\mathrm{mcm}^{-1}\end{array}$ \\
\hline 9219.27 & 9219.29 & Si I & 0.33 & 0.35 & 89 & 85 \\
\hline 9223.34 & 9223.44 & $\mathrm{Ca} \mathrm{I}$ & 0.37 & 0.42 & 23 & 17 \\
\hline 9225.99 & $?$ & $?$ & 0.2 & 0.40 & 8 & 9 \\
\hline 9226.94 & 9226.90 & $\mathrm{Na} \mathrm{I}$ & 0.35 & 0.41 & 28 & 12 \\
\hline 9229.97 & $?$ & $?$ & / & 0.24 & $<6$ & 9 \\
\hline 9231.30 & 9230.792 & He I & $>0.52$ & I & 30 & $<11$ \\
\hline 9232.88 & 9232.83 & Ti I & 0.38 & 0.43 & 36 & 27 \\
\hline 9233.58 & 9233.56 & Si I & 0.40 & 0.41 & 252 & 240 \\
\hline 9238.25: & 9238.17 & Cr I & 0.31 & 0.29 & 36: & 30 \\
\hline 9238.60: & $?$ & $?$ & 0.30 & 0.30 & 62 & 54 \\
\hline
\end{tabular}

Notes. Table with the measured absorption lines in the spectra of the two NGC2808 stars in the region shown in Fig. 3 . The measured wavenumber (column 1) and the expected wavenumber (Col. 2), as well as the line width for the two stars (Cols. 4 and 5) are given in $\mathrm{cm}^{-1}$. The equivalent widths (Cols. 6 and 7 ) are in $\mathrm{mcm}^{-1}$. For all lines, a gaussian approximation was used.

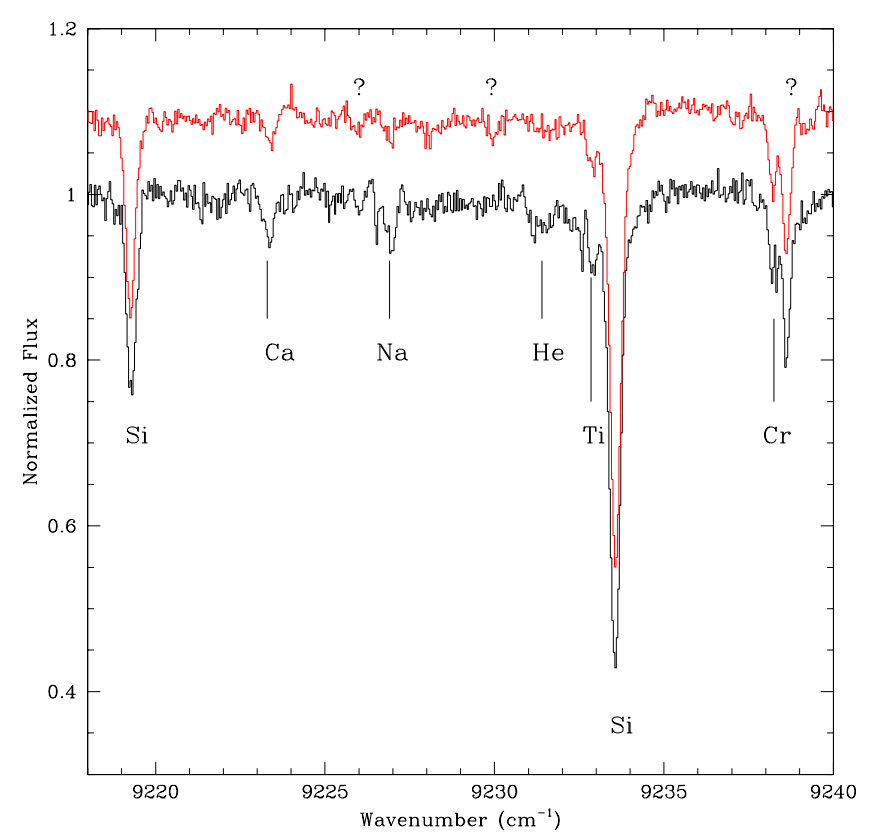

Fig. 5. Spectra of the targets: star 46422 (top - red), star 48889 (bottom - black). All the lines listed in Table 2 are indicated. The question mark indicates lines measured but with no element identification.

There is good agreement between the equivalent widths of the lines of the two stars, but most lines appear slightly stronger in the spectrum of star 48889 . This likely indicates that 48889 is slightly cooler than 46422 . The line at 9231.3 is absent in the spectrum of star 46422 . We also note that all the other lines have line widths not larger than $12 \mathrm{~km} \mathrm{~s}^{-1}\left(0.4 \mathrm{~cm}^{-1}\right)$, while the 9231.3 feature is substantially wider. Close to the 9231.3 line towards the blue there is a line at $\sim 9232.88 \mathrm{~cm}^{-1}$ that is stronger in star 48889 . This line could in principle be identified with a Si line at $9233.10 \mathrm{~cm}^{-1}$, but the frequency of the transition agrees very well with a Ti line at $9232.83 \mathrm{~cm}^{-1}$. This line is sometimes seen in absorption in the spectra of cool M stars (O'Brien \& Lambert 1986). O'Brien \& Lambert (1986) also mention an unidentified line at $10831.3 \AA$ (or $9229.975 \mathrm{~cm}^{-1}$ ) in the spectra of stars later than spectral class $\mathrm{M}$, with an equivalent width of $16 \mathrm{~m} \AA$ in $\alpha$ Her. This line is visible in star 46422 with an equivalent width of $9 \mathrm{mcm}^{-1}$, and we can set an upper limit of $6 \mathrm{mcm}^{-1}$ for star 48 889; our targets are hotter and fainter than $\alpha$ Her and in addition have a rather low metallicity, which would naturally decrease the strength of most lines.

Finally, we consider the possibility that the 9131.3 feature is the blend of two so far unidentified lines. In principle, it can be reproduced with two lines, one at $\sim 9131.15$ and one at $\sim 9131.6 \mathrm{~cm}^{-1}$, each with an equivalent width of about $15 \mathrm{mcm}^{-1}$. We are not aware of known or reported lines at these wavenumbers; possible nearby candidates are two $\mathrm{Si}$ lines $(9131.81,9131.32)$. However, we note that the difference between the two stars for the two lines would be very large, whereas a few Si lines we checked in the UVES spectra (6125.021, 6142.483 $\AA$ ) agree to within $2 \mathrm{~m} \AA$ in their equivalent widths. In addition, if the observed feature were the product of the blend of the two Si lines, one may also expect companion lines at 9130.42 and at $9233.10 \mathrm{~cm}^{-1}$, which are not observed (as discussed above; note that the bluer would be partially blended with the strong 9233.56 line).

The combination of the large equivalent width of the line, the missing companion Si lines, that no lines have been reported at this wavenumber in the literature (even for more metal rich and/or cooler stars) and that the observed feature qualitatively agrees with what is expected a priori, let us conclude that the 9131.3 line is due to He, although we cannot exclude a priori that it is a blend of two unknown lines.

\section{He abundance difference}

We estimated the difference in He abundance using our chromospheric models. The Ca II $\mathrm{K}$ line core profiles in Fig. 6 show that the star 48889 was slightly more active than star 46422 , but the difference is not so dramatic as to require the use of a completely different model. This difference is compatible with a low level of chromospheric variability.

The basic models are those for star 48889 developed in Mauas et al. (2006), which have only been modified in the upper chromosphere and made slightly hotter to take into account the higher chromospheric activity level of this star in 2010 with respect to 2004. However, this update does not involve a substantial change from the previous model. No velocity fields are considered for these simulations. Maintaining the same $T$ versus (vs.) $\log (m)$ distribution, we self-consistently recomputed the non-LTE radiative transfer and the statistical and hydrostatic equilibrium equations, assuming different values of the $\mathrm{He}$ abundance. Using the program Pandora (Avrett \& Loeser 1984), in 


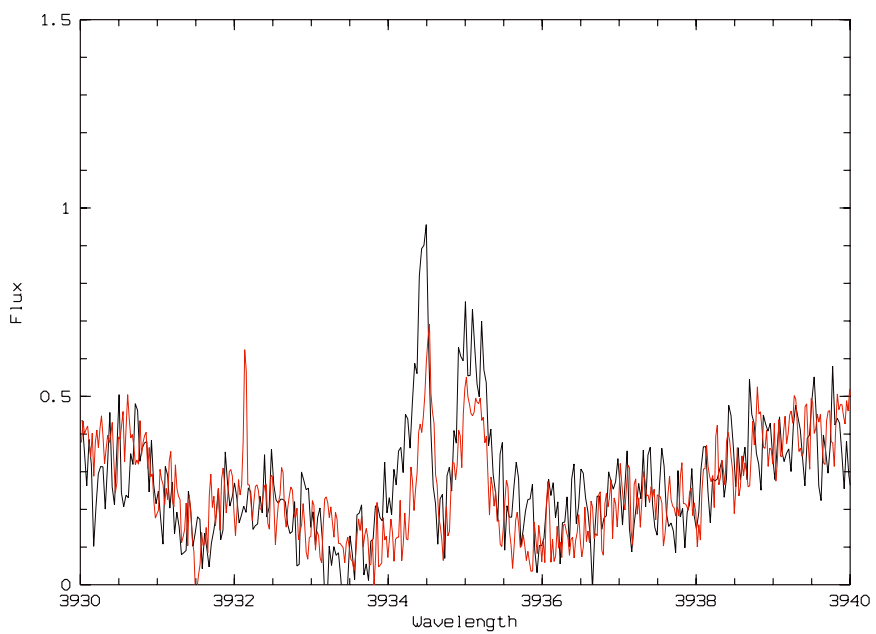

Fig. 6. Ca II K line core spectra of the targets; star 48889 (black) is slightly more active than star 46422 (red), as the core emission is slightly higher.

each case we computed non-LTE populations for 10 levels of H, 9 of C I (see Mauas et al. 1990), 15 of Fe I, 8 of Si I (see Cincunegui \& Mauas 2001), 8 of $\mathrm{Ca}$ I and Na I, 6 of Al I (see Mauas et al. 2002), and 7 of Mg I (see Mauas et al. 1988). In addition, we computed 6 levels of $\mathrm{He}$ II and $\mathrm{Mg}$ II, and 5 of $\mathrm{Ca}$ II. For every species under consideration, we included all the bound-free transitions and the most important bound-bound transitions. The Ly- $\alpha, \mathrm{Ca}$ II $\mathrm{H}$ and $\mathrm{K}$, and $\mathrm{Mg}$ II $h$ and $k$ lines were all computed with a full partial-redistribution treatment (for a discussion, see Falchi \& Mauas 1998). For He I, we used the 29-level atomic model used by Mauas et al. (2005) and Andretta et al. (2008).

It is well known that a strong coronal radiation can ionize $\mathrm{He}$, and enhance the strength of the line via recombination to its higher levels (for a discussion of this mechanism in the Sun, see Mauas et al. 2005). However, these stars are well beyond the so-called coronal "dividing line" (Linsky \& Haisch 1979; Maggio et al. 1990), and are therefore by far too cool and too luminous to possess a corona. The only coronal activity detected in metal poor stars is in short-period binary systems (Pasquini et al. 1991), which is not the case for our sample stars. Furthermore, O'Brien \& Lambert (1986) found that this effect is not important for giant stars. The resulting spectra were broadened by a Gaussian of $17 \mathrm{~km} \mathrm{~s}^{-1}$ FWHM.

Figure 7 shows the results of the modeling: star 46422 spectrum is compatible with a non detection. The figure shows the model with $Y \sim 0.22$, that has an equivalent width close to the upper limit in Table 2 . We consider this value a valid upper limit and the model a good representation of the observed spectrum. An extra absorption in this area seems to be present, so at face value a model with no He line would not be fully compatible with the spectrum. However, given the uncertainties in the He line width and the critical placement of the continuum, a claim of He detection for this star would be an overinterpretation of the data.

For star 48889 , an abundance of $Y \geq 0.39$ is needed to reproduce the observed spectrum. We have tried different values of $Y$, and the model line profile would still be compatible with the observed one for a $\mathrm{He}$ abundance as high as $Y \sim 0.5$ in this star. When considering all the uncertainties involved, as discussed in the previous section and the uncertainty in the placement of the continuum, we infer that our $Y=0.39$ model is a lower limit for

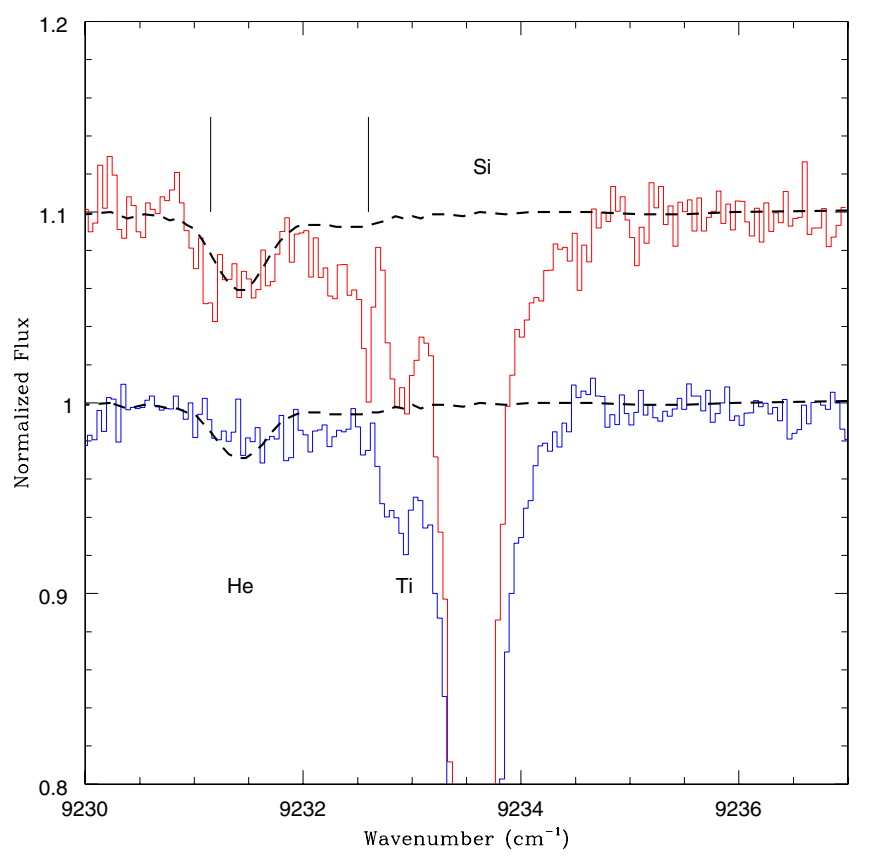

Fig. 7. Observed spectra vs. He models. The He lines in the models have been shifted by $17 \mathrm{~km} \mathrm{~s}^{-1}$. The model superimposed on the data for star 46422 (lower spectrum) has $Y=022$. We find that this model is the most He-rich still compatible with the observed spectrum (see text). For star 48889 (upper spectrum), the model has $Y=0.39$. This is the minimum $\mathrm{He}$ abundance compatible with the observed spectrum, but a higher value of $Y$ cannot be excluded. Observed spectra are continuous lines, where the models are dashed (black) lines. Other spectral lines are marked. The position of the telluric lines in the spectrum of star 48889 is marked as vertical bars above the spectra.

this star. Since we aim to derive relative He abundances rather than absolute values that are affected by much larger uncertainties, our models show that a difference of at least $\Delta Y=0.17$ is needed to reproduce the observed spectra.

Such a large difference in He abundance would increase the opacity, therefore modifying the spectra of these He-rich stars. This problem was studied by Bohm Vitense (1979), who pointed out that the effect is an increase in the gravity of the stars, as quantitatively addressed by Pancino et al. (2011). A helium abundance increase by $\Delta Y=0.10$ (from 0.25 to 0.35 ) in subgiant stars leads to a higher metallicity by $\Delta[\mathrm{Fe} / \mathrm{H}]=0.08$ dex, based on a simplified treatment of the atmosphere where the increased helium abundance is approximated by an artificial increase in the gravity. A more refined atmospheric modelling finds a smaller metallicity increase, i.e. $\Delta[\mathrm{Fe} / \mathrm{H}] \simeq 0.02-0.03 \mathrm{dex}$ (Pancino, priv. comm.). If we apply the Gray (2008) approximation to our star, a helium increase of $\Delta Y=0.17$ would correspond to a $\Delta \log g=0.06$, that in turn leads to increase the metallicity of about 0.01 and 0.03 dex for FeI and FeII, respectively (Carretta, priv. comm.). This difference is much smaller than the typical error in the metallicity determination of $0.10-0.15 \mathrm{dex}$ estimated by Carretta et al. (2004), who derive essentially the same values of $[\mathrm{Fe} / \mathrm{H}]$ within the errors for the two stars (see Table 1).

As we pointed out in Sect. 2.2, it is not possible to modify the He profiles by changing only the upper layers of the atmosphere. Modifying the atmosphere above around $9000 \mathrm{~K}$ does not change the He profiles, because the source function of the lines is completely determined by the conditions at lower heights. This was mentioned in Mauas et al. (2006), where we 
pointed out that, although the center of $\mathrm{H} \alpha$ is formed at around $12000 \mathrm{~K}$ (see Fig. 2 of Mauas et al. 2006), the computed profiles are unaffected by the structure of the regions where the temperature is higher than $10^{4} \mathrm{~K}$. The profile of the He 10830 , which as in the case of $\mathrm{H} \alpha$ is dominated by radiation, is also unaffected by changes high in the atmosphere. On the other hand, the chromospheres of these stars are, of course, far from being semi-static and homogeneous, as our models assume. We do not have a realistic way to evaluate the inhomogeneities of the chromosphere of these stars in any meaningful way. However, to the best of our present knowledge, we believe that a He abundance difference is the simplest and most plausible explanation of the observational evidence.

\section{Summary and conclusions}

We have studied two RGB stars in the GC NGC 2808, that have very similar astrophysical parameters but very different $\mathrm{Na}$ and $\mathrm{O}$ abundances. These stars are assumed to represent the initial sub-population with primordial $\mathrm{Na}, \mathrm{O}$, and $\mathrm{He}$ abundances, and a subsequent subpopulation formed out of processed material from the previous population, which is hence $\mathrm{Na}$ and $\mathrm{He}$ enriched and $\mathrm{O}$ depleted. Whereas $\mathrm{Na}$ and $\mathrm{O}$ abundances are available for these and other stars in NGC 2808, the He abundance has never been determined before. Since helium differences are supposed to be responsible for peculiar observed features of the CMD such as the multiple main sequences and the HB morphology, the empirical detection of a He difference would provide the essential and final confirmation of this scenario.

Helium is barely detectable in cool stars, the only observable line being the He 10830 chromospheric line. This line forms in non-LTE conditions and should not be used to derive absolute He abundances, although with the help of a suitable chromospheric model, reliable quantitative information about relative abundances can be obtained under appropriate assumptions. Our study relies on detailed model chromospheres based on highresolution VLT-UVES spectra of the $\mathrm{Ca}$ II $\mathrm{K}$ and $\mathrm{H} \alpha$ lines for the two program stars. These models produce the corresponding He 10830 line profiles for comparison with the observed ones, obtained from VLT-CRIRES high-resolution spectra.

The two program stars have distinctly different features in the spectral region blueward of the He line rest frequency. In particular an absorption line, broader than the other recognized lines, in the spectrum of star 48889 , which is a sodium-rich, oxygen-poor star) is absent in the spectrum of star 46422 (which has normal $\mathrm{O}$ and $\mathrm{Na}$ abundances). We interpret this feature as a blue-shifted $\mathrm{He}$ line and use our models to derive the He abundance differences. The He abundance difference estimated from the models is no less than $\Delta Y=0.17$, in excellent agreement with theoretical predictions based on stellar evolution considerations to account for the observed peculiarities of the $\mathrm{cmD}$. Our results and conclusions are based on the assumption that the two observed stars are so similar, including in terms of their chromospheres, that the same model can be applied to both of them. This assumption relies on there being a strong similarity between their photometric and spectroscopic characteristics. We believe that an abundance difference between both stars is the most natural explanation of the difference in the observed profiles

As a final remark, we also assume that there is no variability between the sets of the infrared and the bracketing optical measurements, hence the chromospheric characteristics that can be modelled by the $\mathrm{Ca}$ II and $\mathrm{H} \alpha$ lines apply also to the He 10830 line. However, variability on different timescales in the He 10830 line was observed in several field giants, e.g. by Lambert \& O'Brien (1986), as pointed out by the referee. Although it cannot in principle be excluded that a significant chromospheric variation may have occurred in star 48889 at the time of the He 10830 observation, which was not monitored by simultaneous optical observations, this variation should not have affected the $\mathrm{Ca}$ II and $\mathrm{H} \alpha$ spectra that bracket the He 10830 spectrum by just -20 and +30 days. This seems rather unlikely to us, and we feel confident that the enhanced He 10830 line in star 48889 is indicative of higher helium abundance rather than of variability. However, future observations should take this possibility into account. The detection of He in GC giant stars still has a very short history, and clearly it will be important to measure the extent to which and on which timescales He 10830 variability occurs in these objects.

Acknowledgements. The authors gratefully acknowledge the use of NSO/Kitt Peak FTS data, produced by NSF/NOAO. We thank E. Pancino and E. Carretta for useful comments on metallicity determination in He-enhanced atmospheres. The comments and suggestions of an anonymous referee contributed to improve the quality of the paper. R. Smiljanic kindly performed the independent measurements of the equivalent widths and suggested that the 9231.3 line might be a blend.

\section{References}

Andretta, V., Mauas, P., Falchi, A., \& Teriaca, L. 2008, ApJ, 681, 650

Avrett, E. H., \& Loeser R. 1984, in Methods in Radiative Transfer, ed. W. Kalkofen (Cambridge Univ. Press), 341

Bohm-Vitense, E. 1979, ApJ, 234, 521

Bonifacio, P., Pasquini, L., Spite, F., et al. 2002, A\&A, 390, 91

Bragaglia, A., Carretta, E., Gratton, R. G., et al. 2010, A\&A, 519, A60

Breckinridge, J. B., \& Hall, D. 1973, Sol. Phys., 28, 15

Cacciari, C., Bragaglia, A., Rossetti, E., et al. 2004, A\&A, 413, 343

Carretta, E., Bragaglia, A., \& Cacciari, C. 2004, ApJ, 610, L25

Carretta, E., Bragaglia, A., Gratton, R. G., et al. 2010, ApJ, 721, L21

Cayrel, R. 1985, in Calibration of fundamental stellar quantities, Dordrecht, 137

Cincunegui, C., \& Mauas, P. J. D. 2001, ApJ, 552, 877

Danks, T., \& Lambert, D. 1985, A\&A, 148, 293

D’ Antona, F., \& Caloi, V. 2008, MNRAS, 390, 693

Decressin, T., Charbonnel, C., Meynet, G., Prantzos, N., \& Ekström, S. 2007, A\&A, 464, 102

Dekker, H., D’Odorico, S., D’Odorico, S., et al. 2000, Proc. SPIE, 4008, 534

Dupree, A., Sasselov, D. D., \& Lester, J. B. 1992, ApJ, 387, L85

Dupree, A. K., Smith, G. H., \& Straeder, J. 2009, AJ, 138, 1485

Dupree, A. K., Straeder, J., \& Smith, G. H. 2011, ApJ, 728, 155

Falchi, A., \& Mauas, P. J. 1998, A\&A, 336, 281

Gratton, R. G., Bonifacio, P., Bragaglia, A., et al. 2001, A\&A, 369, 87

Gratton, R. G., Carretta, E., Bragaglia, A., et al. 2010, A\&A, 517, A81

Gray, D. F. 2008, The Observation and Analysis of Stellar Photospheres (Cambridge: Cambridge University Press)

Jung, Y., \& Bristow, P. 2008, Proc. The 2007 ESO Instrument Calibration Workshop, 225

Käufl, H. U., Ballester, P., Biereichel, P., et al. 2004, Proc. SPIE, 5492, 1218

Linsky, J. L., \& Haisch, B. M. 1979, ApJ, 229, L27

Maggio, A., Vaiana, G. S., Haisch, B. M., et al. 1990, ApJ, 348, 253

Mauas, P. J., Avrett, E. H., \& Loeser, R. 1988, ApJ, 330, 1008

Mauas, P. J., Avrett, E. H., \& Loeser, R. 1990, ApJ, 357, 279

Mauas, P. J., Fernández Borda, R., \& Luoni, M. L. 2002, ApJS, 142, 285

Mauas, P. J. D., Andretta, V., Falchi, A., et al. 2005, ApJ, 619, 604

Mauas, P. J. D., Cacciari, C., \& Pasquini, L. 2006, A\&A, 454, 609

Moehler, S., Dreizler, S., Lanz, T., et al. 2007, A\&A, 475, L5

O’ Brien, G. T., \& Lambert, D. L. 1986, ApJS, 62, 899

Pancino, E., Mucciarelli, A., Sbordone, L., et al. 2011, A\&A, 527, 18

Pasquini, L., Fleming, T., Spite, F., \& Spite, M. 1991, A\&A, 249, L23

Piotto, G., Villanova, S., Bedin, L. R., et al. 2005, ApJ, 621, 777

Piotto, G., Bedin, L., Anderson, J., et al. 2007, ApJ, 661, L53

Smith, G. H., Dupree, A. K., \& Straeder, J. 2004, PASP, 116, 819

Stetson, P. B., \& Pancino, E. 2008, PASP, 120, 1332

Wallace, L., Hinkle, K., \& Livingston, W. 1993, An Atlas of the Photospheric Spectrum from 8900 to $13600 \mathrm{~cm}^{-1}$ (7350 to $11230 \AA$ A), N.S.O. Technical Report \#93-001

Zirin, H. 1982, ApJ, 665, 669 